FTPI-MINN-18/06, UMN-TH-3715/18

\title{
Supersymmetric Tools in Yang-Mills Theories at Strong Coupling: the Beginning of a Long Journey ${ }^{1}$
}

\author{
Mikhail Shifman \\ William I. Fine Theoretical Physics Institute, University of Minnesota, \\ Minneapolis, MN 55455, USA
}

\begin{abstract}
Development of holomorphy-based methods in super-Yang-Mills theories started in the early 1980s and lead to a number of breakthrough results. I review some results in which I participated. The discovery of Seiberg's duality and the Seiberg-Witten solution of $\mathcal{N}=2$ Yang-Mills were the milestones in the long journey of which, I assume, much will be said in other talks. I will focus on the discovery (2003) of non-Abelian vortex strings with various degree of supersymmetry, supported in some four-dimensional Yang-Mills theories and some intriguing implications of this discovery. One of the recent results is the observation of a soliton string in the bulk $\mathcal{N}=2$ theory with the $U(2)$ gauge group and four flavors, which can become critical in a certain limit. This is the case of a "reverse holography," with a very transparent physical meaning.
\end{abstract}

\footnotetext{
${ }^{1}$ Based on the talk at the Dirac Medal Award Ceremony, ICTP, March 22, 2017.
} 
I should say that receiving the Dirac Medal is the highest honor for me as a theoretical physicist. I am deeply grateful to Abdus Salam Center for Theoretical Physics for awarding me this highly prestigious Prize.

\section{Introduction}

So far quantum field theory (QFT) remains the basis of our understanding of fundamental laws of Nature. QFT is ninety years old. If you look at books and reviews devoted to QFT written in the 1950s and '60s and compare them with today's reviews, you will hardly say this is one and the same discipline. Many questions on which we focus at present could not even be formulated then.

Since then QFT underwent two dramatical changes: the discovery and development of Yang-Mills theories which, as we know, run our four-dimensional world, and the discovery and development of supersymmetric gauge theories which dominate the modern theoretical landscape. The first four-dimensional supersymmetric theory - supergeneralization of quantum electrodynamics - was constructed in 1970 and published in [1] by Golfand and Likhtman. The breakthrough works of Wess and Zumino paved the way to remarkable advances in this area which continue unabated forty years later.
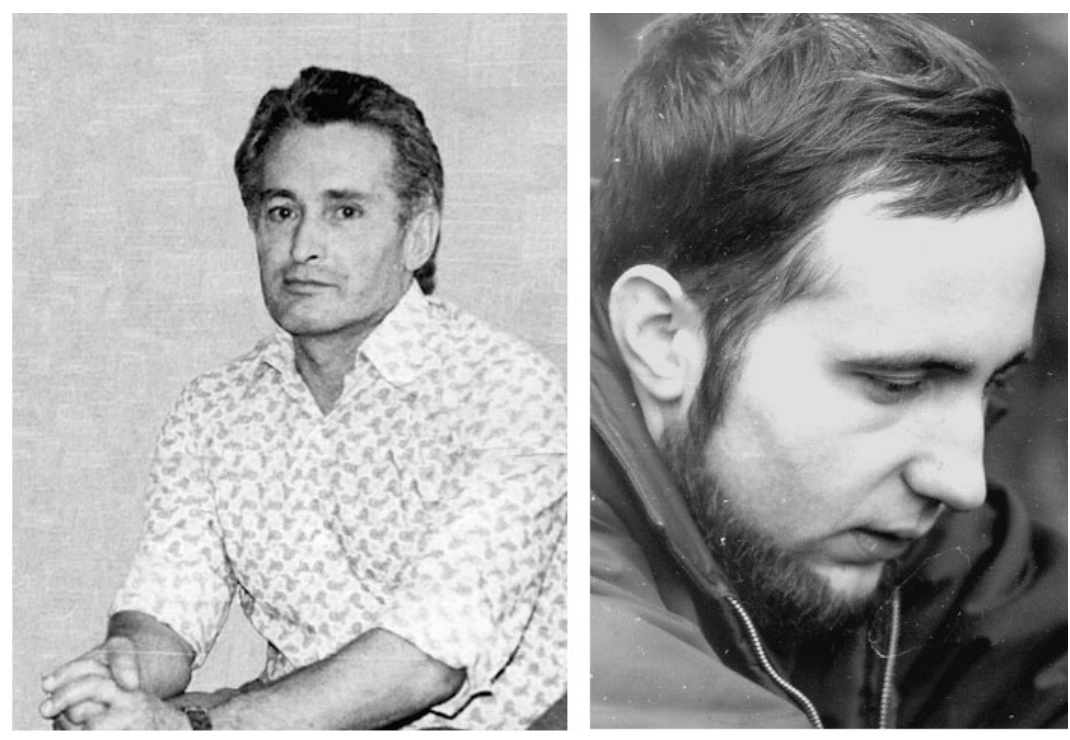

Figure 1: Yury Golfand and Evgeny Likhtman, circa 1980.

Although none of the expected superpartners have been experimentally detected 
so far supersymmetry changed quantum field theory at a deep level by providing answers to some hard questions, such as divergences in the vacuum energy density. Any theory which can be embedded in $\mathcal{N}=4$ super-Yang-Mills is ultraviolet finite.

The first exact statement of this type was the vanishing of the vacuum energy in supersymmetric theories ${ }^{2}$ Endowing Yang-Mills theories with supersymmetry creates a broad class of the so-called protected quantities which can be calculated exactly in both weak and strong coupling regimes. In 1982-83 we observed [4] that not only in the vacuum, but in all backgrounds which conserve a part of supercharges exact results are attainable. Analysis of instantons [5] in super-Yang-Mills theories [4.6] was the beginning of a long journey on which we embarked and of which I am going to speak today. I will only briefly summarize "old" results since they were reviewed in detail in my 1999 Sakurai talk [7]. Then I will pass to a new era which to my mind is associated with the breakthrough solution of slightly deformed $\mathcal{N}=2$ super-Yang-Mills theory by Seiberg and Witten [8].

\section{Setting the stage (1980s) and later}

\subsection{Instantons and $\beta$ functions in four and two dimensions}

The instanton background field conserves half of the supercharges. The other half acts on instanton nontrivially producing fermion zero modes. The number of the instanton zero modes is related to the number of nontrivially realized symmetries. The supercharges that are conserved guarantee that all quantum corrections cancel, and hence the instanton measure can be exactly calculated, impying, in turn, the exact $\beta$ functions. In pure super-Yang-Mills theories with various degree of supersymmetry we obtain

$$
\beta(\alpha)=-\left(n_{b}-\frac{n_{f}}{2}\right) \frac{\alpha^{2}}{2 \pi}\left[1-\frac{\left(n_{b}-n_{f}\right) \alpha}{4 \pi}\right]^{-1},
$$

where $n_{b}$ and $n_{f}$ count the gluon and gluino zero modes, respectively. For $\mathcal{N}=1$ we have $n_{b}=2 n_{f}=4 T_{G}$ where $T_{G}$ is the dual Coxeter number (it is also called $1 / 2$ the Dynkin index; for $S U(N)$ we have $\left.T_{G}=N\right)$. For $\mathcal{N}=2$, one gets $n_{b}=n_{f}=4 T_{G}$,

\footnotetext{
${ }^{2}$ As early as in 1950s, Wolfgang Pauli delivered a landmark series of lectures at the Swiss Federal Institute of Technology (ETH) in Zurich. They were published in English by MIT Press only in 1973. In Section 9 of Volume 6 2 Pauli discusses the vacuum energy density in various field theories known at that time. He observes that adding the Dirac spinor contribution to that of two complex scalar fields cancels divergences and produces zero vacuum energy - the first ever hint to supersymmetry! In the context of supersymmetric field theories the zero vacuum energy was first observed in 3 .
} 
implying that the $\beta$ function reduces to one-loop. For $\mathcal{N}=4$ the $\beta$ function vanishes since $n_{f}=2 n_{b}$.

The main lesson obtained in [4] was as follows. Equation (1] makes explicit that all coefficients of the $\beta$ functions in pure super-Yang-Mills theories have a geometric origin since they are in one-to-one correspondence with the number of symmetries nontrivially realized on instantons.

In theories with matter, apart from the gluon and gluino zero modes, one has to deal with the zero modes of the matter fermions. While the gluon/gluino $Z$ factors are related to the gauge coupling constant $g^{2}$ itself, this is not the case for the $Z$ factors of the matter fermions. Therefore, in theories with matter the exact instanton measure implies an exact relation between the $\beta$ function and the anomalous dimensions $\gamma_{i}$,

$$
\beta(\alpha)=-\frac{\alpha^{2}}{2 \pi}\left[3 T_{G}-\sum_{i} T\left(R_{i}\right)\left(1-\gamma_{i}\right)\right]\left(1-\frac{T_{G} \alpha}{2 \pi}\right)^{-1},
$$

where $T\left(R_{i}\right)$ is the Dynkin index in the representation $R_{i}$,

$$
\operatorname{Tr}\left(T^{a} T^{b}\right)=T\left(R_{i}\right) \delta^{a b},
$$

and $T^{a}$ stands for the generator of the gauge group $G$ in the appropriate representation which can be arbitrary.

Equation (2) is valid for arbitrary Yukawa interactions of the matter fields. The Yukawa interactions show up only through the anomalous dimensions. It appeared first in [9], and shortly after in a more closed form in $[10]^{3}$ The latter work presented the solution of the anomaly supermultiplet problem by virtue of the Wilsonean approach, more of which will be said in Arkady Vainshtein's talk. Recently the anomaly supermultiplet problem was revisited [12] and extended to a number of theories which were not considered in the 1980s.

Equation (2) played a crucial role in establishing the edges of the conformal window in Seiberg's duality $[13]$.

By the same token exact $\beta$ functions can be obtained and the geometric nature of the coefficients revealed in two-dimensional sigma models. In the 1980s this was done for the $\mathcal{N}=(2,2)$ sigma models [14], i.e. the model with extended supersymmetry. Recently an interesting class of "heterotic" $\mathcal{N}=(0,2)$ sigma models was discovered in connection with non-Abelian strings which will be discussed later. It turns out that the chiral $\mathcal{N}=(0,2)$ sigma models (in which there is no symmetry between

\footnotetext{
${ }^{3}$ For the current status of direct perturbative derivations of the NSVZ $\beta$ function see [1] and references therein.
} 
the left- and right-moving fermions) represent a direct analog of four-dimensional Yang-Mills. This was an exciting finding.

Thus, in the minimal heterotic $C P(1)$ model, in which all left-handed fermions are dropped ${ }^{4}$ we have 17

$$
\beta\left(g^{2}\right)=-\frac{g^{4}}{4 \pi} \frac{1}{1-\frac{g^{2}}{4 \pi}},
$$

which is a direct analogue of Eq. (1) in super-Yang-Mills. In this particular $C P(1)$ model with $\mathcal{N}=(0,2)$ supersymmetry one can introduce "matter," i.e. $N_{f}$ superfields of $\mathcal{N}=(0,2)$ supersymmetry which contain only a single left-moving fermion degree of freedom (per flavor). The fermion state in this multiplet has no bosonic counterpart - other superfield components are auxiliary.

In this model, instead of (3) we arrive at 17

$$
\beta\left(g^{2}\right)=-\frac{g^{4}}{4 \pi} \frac{1+\frac{N_{f} \gamma}{2}}{1-\frac{g^{2}}{4 \pi}},
$$

where $\gamma$ is the anomalous dimension of the "matter" superfield. This expression is similar to that in Eq. (2). The only difference is that the "matter" field fermions do not show up in the numerator at one loop; they appear only in the second and higher loops. This is a special feature of the two-dimensional $C P(N-1)$ models.

If $N_{f}=1$ then $\mathcal{N}=(0,2)$ is uplifted to $(2,2)$ and the $\beta$ function in (4) becomes one-loop. The numerator in (4) is exactly canceled by the denominator. At sufficiently large $N_{f}$ Eq. (4) obviously exhibits an infrared fixed point. This is similar to the Banks-Zaks phenomenon [18]. Therefore, in the minimal heterotic $(0,2) C P(1)$ model a conformal window exists. The question of whether a dual representation exists in this model is still open. I think this is a very challenging question.

The above results were extended to $C P(N-1)$ with $N>2[19]$. To this end the minimal model could not be used, as was mentioned above: a quantum anomaly makes it inconsistent. However, the non-minimal $\mathcal{N}=(0,2)$ model discovered in the studies of non-Abelian vortex-strings supported in $\mathcal{N}=1$ super-Young-Mills [20] is selfconsistent for any $N$. The non-minimal model is obtained from the conventional $(2,2)$ model by deforming it by an extra right-moving field $\zeta$ with a heterotic coupling parametrized by a constant $h$. In this case we derived [19] the exact relation between the $\beta$ function and the anomalous dimensions $\gamma$ of the "matter" fields,

$$
\beta_{g}=-\frac{g^{2}}{4 \pi} \frac{N g^{2}\left(1+\gamma_{\psi_{R}} / 2\right)-h^{2}\left(\gamma_{\psi_{R}}+\gamma_{\zeta}\right)}{1-\left(h^{2} / 4 \pi\right)},
$$

\footnotetext{
${ }^{4}$ The minimal models of this type do not exist for $C P(N-1)$ with $N>2$ because of the anomaly 15, 16.
} 
where $\gamma_{\psi_{R}}, \gamma_{\zeta}$ are the anomalous dimensions of the $\psi_{R}, \zeta_{R}$ fields, respectively. Here $g^{2}$ is the constant parametrizing the $C P(N-1)$ target space, $h$ is a heterotic deformation parameter, and $\zeta_{R}$ is an additional field breaking $(2,2)$ down to $(0,2)$. It has no left-moving counterpart. Equation (5) has the same structure as that in $\mathcal{N}=1$ Yang-Mills with matter, see (2).

\section{$2.2 \quad$ Adler functions}

The above ideas that allowed us to obtain the exact $\beta$ functions could have been used in the 1980s to obtained exact predictions for the Adler $D$ functions in SQCD. This never happened, however. Only in 2015 this gap was filled [21]. The Adler function is defined as an infinite set of the diagrams symbolically depicted in Fig. 2.

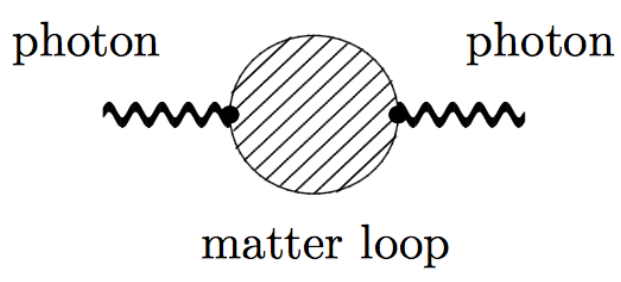

Figure 2: The Adler function in $\mathcal{N}=1$ SQCD. The shaded circle presents all loop graphs. Borrowed from the second paper in [11].

The exact result is

$$
D\left(Q^{2}\right)=\frac{3}{2} N_{c} \sum_{f} q_{f}^{2}\left[1-\gamma\left(\alpha_{s}\left(Q^{2}\right)\right)\right]
$$

where the sum runs over all flavors, $q_{f}$ is the electric charge of a given flavor, $\gamma$ is the anomalous dimension of the matter fields. It is the same for all matter fields assuming that they all belong to the fundamnental representation of color. Moreover, $\alpha_{s}$ is the strong coupling constant. The result is plotted in Seiberg's conformal window $\frac{3}{2} N_{c}<N_{f}<3 N_{c}$ in Fig. 3 .

For a very recent discussion of Eq. (6) in perturbation theory I refer the reader to [22]. This latter paper is based on a development in supersymmetric perturbation theory which I'd like to mention [23]. It is known under the name the "NSVZ regularization scheme," with higher derivatives. We anticipated the existence of such a scheme in the 1980s. It took 30 years to develop it and make it work! 


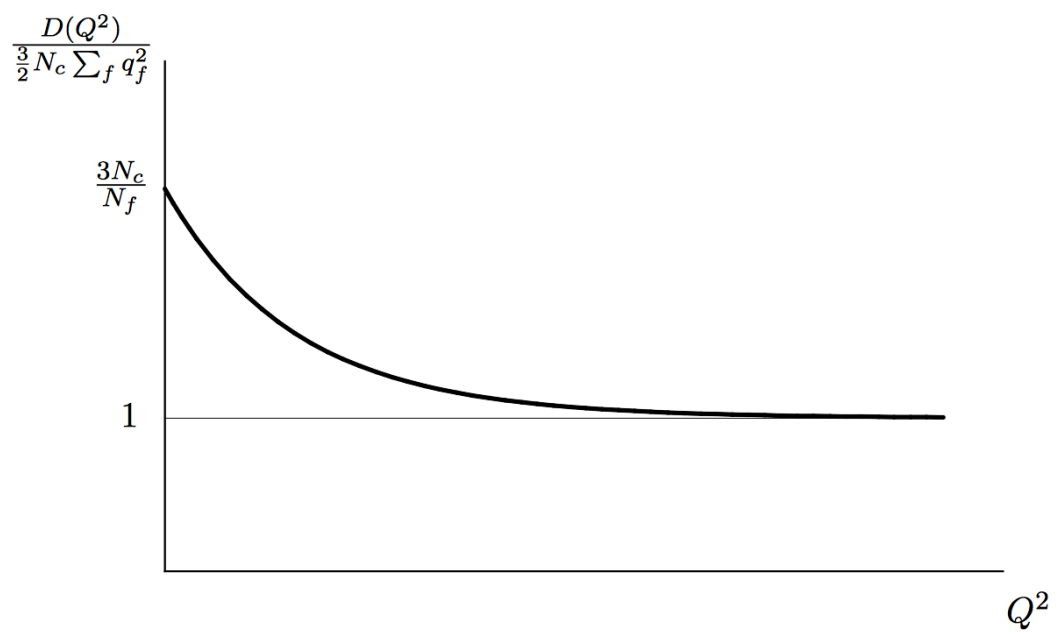

Figure 3: $D\left(Q^{2}\right)\left(\frac{3}{2} N_{c} \sum_{f} q_{f}^{2}\right)^{-1}$ versus $Q^{2}$. The horizontal lines corresponds to $N_{f}=3 N_{c}$, i.e. the right edge of the conformal window.

\subsection{Gluino condensate and localization}

Simultaneously with the first exact $\beta$ functions we found a way to calculate the gluino condensate 24

$$
\mathcal{O}_{\lambda}=\operatorname{Tr}\left(\lambda^{\alpha} \lambda_{\alpha}\right)
$$

This problem tortured us for at least two years, until we realized that if one considers $n$-point functions of operators which are $Q$-closed (such as $\mathcal{O}_{\lambda}$ ), while their spacial derivatives are $Q$-exact $\left.\right|^{5}$ then the above $n$-point functions must be independent of coordinates. For example, the $S U(2)$ instanton completely saturates the following two-point function:

$$
\left\langle T\left[\mathcal{O}_{\lambda}(x) \mathcal{O}_{\lambda}(0)\right]\right\rangle=c_{s}^{2} \Lambda^{6}, \quad \text { for } \forall x,
$$

in $\mathcal{N}=1$ super-Yang-Mills theory with the $S U(2)$ gauge group. Here $c_{s}$ is an exactly calculable numerical constant and $\Lambda$ is the dynamical scale. The exact gluino condensate follows from (8),

$$
\left\langle\mathcal{O}_{\lambda}\right\rangle= \pm c_{s} \Lambda^{3}
$$

I think we were the first to prove the above theorem (please, correct me if I am wrong). By now it became instrumental in many supersymmetry-based constructions, for instance, in Nekrasov's localization, of which I will say a few words later.

\footnotetext{
${ }^{5}$ In the case of the gluino condensate $\left[\bar{Q}^{\dot{\beta}}, \mathcal{O}_{\lambda}\right]=0$ while $\partial_{\alpha \dot{\beta}} \mathcal{O}_{\lambda} \sim\left\{\bar{Q}^{\dot{\beta}}, \operatorname{Tr}\left(\lambda^{\beta} G_{\beta \alpha}\right)\right\}$.
} 
The calculation of (8) was performed at short distances, $|x| \ll \Lambda^{-1}$, where this correlation function is saturated by instantons of small size, $\rho \sim|x|$. However, the theory itself $-\mathcal{N}=1$ pure Yang-Mills - is strongly coupled. Although the result in (8) was perfectly well-defined mathematically I still could not get rid of a feeling of vague dissatisfaction. It was desirable to revisit the problem at weak coupling (in the Higgs regime), calculate the gluino condensate enjoying full theoretical control over the theory, and then analytically continue back to strong coupling using the known holomorphic behavior of the condensate on the mass parameter $m$ of the Higgs field. On general grounds it was known that in the $S U(2)$ gauge theory $\left\langle\mathcal{O}_{\lambda}\right\rangle \sim \sqrt{m}$. The only singularity is of the square root type - this statement is exact. The simple analytic continuation method we developed in 1985 perhaps can be viewed as a primitive rudimentary precursor to the powerful Seiberg-Witten solution.

The result for the gluino condensate obtained in this way [25] (see also [26]) after taking $m$ to be equal to the ultraviolet cut-off has the same functional dependence as in $(9)$, namely $\left\langle\mathcal{O}_{\lambda}\right\rangle= \pm c_{w} \Lambda^{3}$ where the subscript $w$ means the weak coupling calculation as opposed to $c_{s}$ appearing at strong coupling. Originally it was found [25] that $c_{s}=\sqrt{\frac{4}{5}} c_{w}$. Now there is no doubt (see e.g. 27$]$ ) that it is the weak coupling calculation that produces the correct value of the coefficient in the gluino condensate. A physical explanation of the discrepancy above is not found yet.

The paper [25] was entitled "Supersymmetric Instanton Calculus (Gauge Theories with Matter)." The theory we dealt with to determine the gluino condensate in the weak coupling (Higgs) regime was well-defined at all distances, including large, because of complete Higgsing. Under these circumstances we were certain that $\left\langle\mathcal{O}_{\lambda}\right\rangle$ was saturated by one instanton. It was natural to think that the size of the saturating instanton would be of the order of $\rho \sim v^{-1}$ where $v$ is the vacuum expectation value of the Higgs field. However, a great surprise expected us en route. A remarkable observation was made in [25]: the integral over the instanton size proved to be completely saturated by zero-size instantons, because we represented it as a full derivative which reduced to a delta function, $\delta\left(\rho^{2}\right)$ in the integrand. This was probably the first example of self-localization which later grew into a powerful construction of the Nekrasov localization 28.

\subsection{Mass spectrum}

The first superalgebra in four-dimensional field theory was derived by Golfand and Likhtman [1] in the form

$$
\left\{\bar{Q}_{\dot{\alpha}} Q_{\beta}\right\}=2 P_{\mu}\left(\sigma^{\mu}\right)_{\alpha \beta}, \quad\left\{\bar{Q}_{\alpha} \bar{Q}_{\beta}\right\}=\left\{Q_{\alpha} Q_{\beta}\right\}=0,
$$


i.e. with no central extensions. Possible occurrence of extensions (elements of superalgebra commuting with other generators) was first mentioned in an unpublished paper of Łopuszański and Sohnius [29] where the last two anticommutators were modified as (see also [30])

$$
\left\{Q_{\alpha}^{I} Q_{\beta}^{G}\right\}=Z_{\alpha \beta}^{I G} .
$$

The superscripts $I, G$ mark extended supersymmetry. The central charge derived in this paper was that in $\mathcal{N}=2$ superalgebra in four dimensions, $Z_{\alpha \beta}^{I G} \sim \varepsilon_{\alpha \beta} \varepsilon^{I G}$. It is Lorentz scalar and is relevant to the magnetic monopole (dyon) masses. In the 1980s the focus was on consequences from the Lorentz scalar central charges.

It was generally understood that superalgebras with (Lorentz-scalar) central charges can be obtained from superalgebras without central charges in higher-dimensional space-time by interpreting some of the extra components of the momentum as central charges. If the "central charges" carry Lorentz indices or contain contributions from quantum anomalies (or both), the dimension reduction procedure cannot be used. Above I put "central charges" in the quotation marks because $Z_{\{\alpha \beta\}}$ or $Z_{\mu}$ or other Lorentz-noninvariant elements of superalgebras in various dimensions are not central in the strict sense of the word: they only commute with $Q_{\alpha}, \bar{Q}_{\dot{\alpha}}$ and $P_{\mu}$, and do not commute with Lorentz rotations, since they carry the Lorentz indices. They are associated with extended topological defects — such as domain walls or strings - and could be called brane charges [12]. Leaving this subtlety aside, I will continue to refer to these elements as central charges (CCs), or, sometimes, tensorial central charges.

Why the central extension of $(10)$ is so important? In such theories the so-called short supermultiplets exist. Because they are short their mass (or tension in the case of extended objects) is exactly equal to the corresponding CC [31]. The latter are of topological nature and are exactly calculable in many cases. Thus, centrally extended supersymmetric theories present the first example of four-dimensional QFT in which the physical masses (tensions) are known exactly, even at strong coupling. A great example is the mass formula for monopoles/dyons in the Seiberg-Witten solution [8]. 


\section{1990s and later}

\subsection{Anomalous contribution to central charges}

A mystery of the kink mass in the simplest two-dimensional $\mathcal{N}=(1,1)$ model

$$
\mathcal{L}=\frac{1}{2}\left\{\partial_{\mu} \phi \partial^{\mu} \phi+\bar{\psi} i \not \partial \psi-\left(\frac{\partial \mathcal{W}}{\partial \phi}\right)^{2}-\frac{\partial^{2} \mathcal{W}}{\partial \phi^{2}} \bar{\psi} \psi\right\}
$$

tortured theoretical physicists since 1983 for fifteen years. Here $\phi(t, z)$ is a real scalar field, $\psi$ is a Majorana spinor, and $\mathcal{W}(\phi)$ is a superpotential. Superalgebra of this model is

$$
\left\{Q_{\alpha}, \bar{Q}_{\beta}\right\}=2\left(\gamma^{\mu}\right)_{\alpha \beta} P_{\mu}+2 i\left(\gamma^{5}\right)_{\alpha \beta} \mathcal{Z}
$$

where $\mathcal{Z}$ is the Lorentz-invariant central charge,

$$
\mathcal{Z}=\Delta \mathcal{W} \equiv(\mathcal{W})_{z=+\infty}-(\mathcal{W})_{z=-\infty}
$$

In this model kinks belong to a short BPS-protected multiplet and, therefore, their mass was expected to be exactly equal to $\mathcal{Z}$. However, in numerous calculations (for a review see [32]) this equality was found to be violated already at one loop! The solution was found only in $1998[32]$. It turned out that the equality $M_{\text {kink }}=\Delta \mathcal{W}$ could not be valid because Eq. (14) was incomplete. Nobody suspected the existence of a quantum anomaly which replaces $\Delta \mathcal{W}$ in (14) by

$$
\Delta\left(\mathcal{W}+\frac{1}{4 \pi} \mathcal{W}^{\prime \prime}\right)_{z= \pm \infty}
$$

After this replacement in $(14)$ the amended expression for $\mathcal{Z}$ exactly coincides with the mass $M_{\text {kink }}$ in the short multiplet.

By the way, the model (12) has another anomaly - global [33]. The fermion field in $(12)$ is real. Therefore, the fermion number $F$ is not defined, of course. However, looking at $(12)$ one would say that the fermion parity $(-1)^{F}$ is well defined and conserved. This is a false impression. While conservation of $(-1)^{F}$ is valid in perturbation theory, it is lost nonperturbatively. Say, for a cubic superpotential (leading to a double-well potential) $(-1)^{F}$ ceases to be well-defined for the kink supermultiplet because of a global anomaly discovered in [33]. The short kink supermultiplet consists of a single state for which the fermion parity is neither 1 nor -1 . 


\subsection{The brane charge in $\mathcal{N}=1$ pure Yang-Mills is anomaly}

In Sect. 2.3 I outlined the exact calculation of the gluino condenate carried out in the $1980 \mathrm{~s}$,

$$
2 \operatorname{Tr}\left\langle\lambda^{\alpha} \lambda_{\alpha}\right\rangle=\left\langle\lambda_{\alpha}^{a} \lambda^{a, \alpha}\right\rangle=-6 N \Lambda^{3} \exp \left(\frac{2 \pi i k}{N}\right), k=0,1, \ldots, N-1 .
$$

The above result refers to $\mathcal{N}=1$ super-Yang-Mills. In 1996 Dvali and I found 34 a fascinating application for this result. The $(1,0)$ and $(0,1)$ branes charges below

$$
\left\{Q_{\alpha}, Q_{\beta}\right\}=-4 \Sigma_{\alpha \beta} \bar{Z}
$$

where

$$
\Sigma_{\alpha \beta}=-\frac{1}{2} \int \mathrm{d} x_{[\mu} \mathrm{d} x_{\nu]}\left(\sigma^{\mu}\right)_{\alpha \dot{\alpha}}\left(\bar{\sigma}^{\nu}\right)_{\beta}^{\dot{\alpha}},
$$

are not seen at the classical level in this theory. Nevertheless, they exist [34] as a quantum anomaly. They are saturated by domain walls interpolating between vacua with distinct values of the parameter $k$ in Eq. (16), labeling $N$ distinct vacua of super-Yang-Mills theory with the gauge group $\mathrm{SU}(N)$. The tension of the BPS wall is

$$
T=|Z|=\frac{N}{8 \pi^{2}}\left|\left\langle\operatorname{Tr} \lambda^{2}\right\rangle_{\text {vac } \mathrm{f}}-\left\langle\operatorname{Tr} \lambda^{2}\right\rangle_{\text {vac i }}\right|
$$

where $\mathrm{vac}_{\mathrm{i}, \mathrm{f}}$ stands for the initial (final) vacuum between which the given wall interpolates. This anomaly is in fact a "superpartner" to that in the trace of the energy-momentum tensor.

For the interpolations between the neighboring vacua $\Delta \operatorname{Tr}\left\langle\lambda^{2}\right\rangle$ scales as $N^{0}$. Equation (19) implies then that the wall tension scales as $N^{1}$. Since the string coupling constant $g_{s} \sim 1 / N$, the wall tension is proportional to $T \sim 1 / g_{s}$ rather than $1 / g_{s}^{2}$. Thus, this is not a "normal" soliton but, rather, a D brane. This is the essence of Witten's argument why the above walls should be considered as analogs of D branes 35.

Many interesting consequences ensued. One of them was the Acharya-Vafa derivation of the wall world volume theory $[36]$. Using a wrapped $D$-brane picture and certain dualities they identified the $k$-wall $b^{6}$ world volume theory as $1+2$ dimensional $\mathrm{U}(k)$ gauge theory with the field content of $\mathcal{N}=2$ and the Chern-Simons term at level $N$ breaking $\mathcal{N}=2$ down to $\mathcal{N}=1$. This allowed them to calculate the wall multiplicity.

\footnotetext{
${ }^{6}$ Minimal, or elementary, walls interpolate between vacua $n$ and $n+1$, while $k$-walls interpolate between $n$ and $n+k$.
} 
In fact, even if we consider a given minimal wall, $k_{f}=k_{i}+1$, we deal with several walls, all having one and the same tension. The fact that distinct BPS walls with the same boundary conditions can have one and the same tension is specific for supersymmetry. It was discovered (see [37] and [38,39]) in studies of the BPSsaturated walls - in such walls, even if their internal structures are different, the tension degeneracy is the consequence of the general law $T=|Z|$.

In the field-theoretic setting the $k$-wall multiplicity was derived in [38],

$$
\nu_{k}=C_{N}^{k}=\frac{N !}{k !(N-k) !} .
$$

In particular, for the neighboring walls $k=1$ and hence $\nu=N$. The derivation is based on the fact that the index $\nu$ is topologically stable - continuous deformations of the theory do not change $\nu$. Thus, one can add an appropriate set of matter fields sufficient for complete Higgsing of super-Yang-Mills. Upon Higgsing one can calculate the multiplicity $\nu_{k}$ at weak coupling. It is worth noting that the method suggested in [38, 39] was recently extended [40] to softly broken SQCD at $\theta=\pi$ with the purpose of exploring a newly discovered discrete anomaly [41].

The anomaly similar to (19) was also found in two-dimensional $C P(N-1)$ models [42] (see also [43]). In this case it is a bona fide central charge, since the domain wall reduced to two dimensions becomes a kink (i.e. a localized particle). Since the twodimensional $C P(N-1)$ models with various degree of supersymmetry occur on the world sheet of non-Abelian strings (see below) this anomaly was extensively used in explorations of the non-Abelian strings [44]. I will say more on this in the subsequent sections.

\subsection{Planar equivalence between $\mathcal{N}=1$ super-Yang-Mills and its non-supersymmetric daughters}

In connection with the success of the exact predictions in supersymmetric theories a question was raised as to whether one can draw quantitative lessons for nonsupersymmetric theories. The genesis of this question and first advances are described in detail in the review paper [45]. In 2003 we noted and proved that $\mathcal{N}=1$ superYang-Mills has two orientifold daughters which are perfectly similar to the parent supersymmetric theory except they are not supersymmetric! Namely, the gluino field (described by a Weyl fermion in the adjoint representation) can be replaced by a Dirac fermion in the two-index representation of $S U(N)$ - either symmetric or

antisymmetric. In the large- $N$ limit all correlation functions in the common sector 
in these two theories are equal [46]. In practice, of most interest is the antisymmetric two-index representation because in the $N=3$ case $\mathcal{N}=1$ super-Yang-Mills' daughter is just "our" conventional QCD with one flavor.

Using this fact and extrapolating from $N=\infty$ to $N=3$ we were able to obtain the first analytic prediction for the quark condensate [47] which agrees well with experimental data and lattice simulations.

The "new" large- $N$ limit (the "ASV" limit) in QCD with the two-index antisymmetric fermions (alternative to that of 't Hooft) was rather extensively applied to QCD phenomenology, see e.g. [48]. I want to mention that in the ASV limit, unlike the 't Hooft limit, the widths of exotic mesons, such as tetraquarks, die off at large $N$ [49].

\section{Non-Abelian strings}

Since 2003 I work with Alexei Yung on making relatively realistic theoretical constructions addressing QCD-type confinement based on supersymmetric results and inspired by the Seiberg-Witten solution [8]. As Seiberg and Witten, Alexei and I started from consideration of $\mathcal{N}=2$ Yang-Mills theories in four dimensions, which may or may not be deformed by breaking parameters down to $\mathcal{N}=1$. Unlike [8] our main focus was on the so-called quark vacua.

In this section I will present a general idea of what is now known as non-Abelian strings and some key results such as the exact $2 \mathrm{D}-4 \mathrm{D}$ correspondence. This theme is still very much "work in progress." Therefore, a more complete summary will appear later.

Non-Abelian string is a vortex type soliton supported in four-dimensional YangMills theories. In a class of such theories flux tube solutions were found in the 1980s, but they are not non-Abelian. So is the string in the Seiberg-Witten solution at small deformations $\mu$ (and only at small $\mu$ the solution is possible). All the above vortex strings are similar to the Abrikosov-Nilsen-Olesen string [50]. They are formed at low energies by "photons" after electric charge condensation. All other gauge bosons acquire large masses and do not play a direct role in the string formation.

By non-Abelian string we mean a vortex soliton the structure of which is determined on equal footing by all gauge bosons existing in the bulk (i.e. four-dimensional) theory. Correspondingly, there are no singled out $U(1)$ directions. As a result, new type of moduli on the string world sheet appear, reflecting arbitrariness of "color" orientations in the bulk gauge group $7^{7}$ see Fig. 4. The orientational moduli, combined

\footnotetext{
${ }^{7}$ Two-dimensional quantum oscillations may and usually do provide a mass gap to these moduli
} 
with the conventional translational moduli (Fig. 5), form a dynamically nontrivial sigma model on the string world sheet. As we will see shortly, studying dynamics in two dimensions one can project two-dimensional results for protected quantities onto four dimensions.

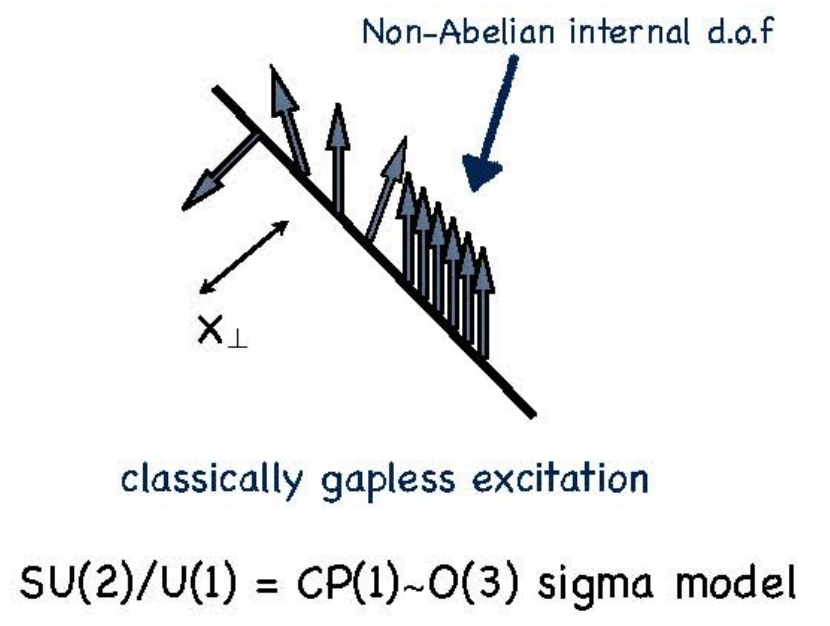

Figure 4: Orientational moduli on the string world sheet, see Sect. 4.2 .

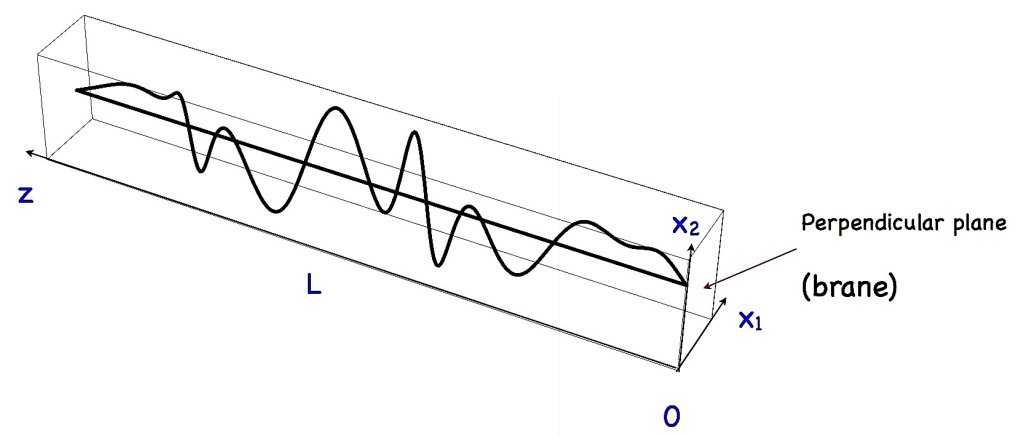

Figure 5: Excitations of translational moduli described by the Nambu-Goto action.

which we refer to as "orientational." 


\subsection{Basic Model}

Chronologically first (and still most convenient) are the bulk $\mathcal{N}=2$ Yang-Mills theories with the gauge group $U(N)$ and $N_{f}=N\left(N_{f}\right.$ is the number of flavors), and the Fayet-Iliopoulos term. The Higgs branch is lifted by the Fayet-Iliopoulos parameter $\xi \neq 0$, and the quark vacua are isolated [51].

The simplest and most pedagogical example is $N=N_{f}=2$. The bosonic part of the theory has the form

$$
\begin{aligned}
S & =\int d^{4} x\left[\frac{1}{4 g_{2}^{2}}\left(F_{\mu \nu}^{a}\right)^{2}+\frac{1}{4 g_{1}^{2}}\left(F_{\mu \nu}\right)^{2}+\frac{1}{g_{2}^{2}}\left|D_{\mu} a^{a}\right|^{2}+\frac{1}{g_{1}^{2}}\left|\partial_{\mu} a\right|^{2}\right. \\
& \left.+\left|\nabla_{\mu} q^{A}\right|^{2}+\left|\nabla_{\mu} \overline{\tilde{q}}^{A}\right|^{2}+V\left(q^{A}, \tilde{q}_{A}, a^{a}, a\right)\right] .
\end{aligned}
$$

Here $\nabla_{\mu}$ is the covariant derivative in the fundamental representation of $S U(2)$, and

$$
\nabla_{\mu}=\partial_{\mu}-\frac{i}{2} A_{\mu}-i A_{\mu}^{a} T^{a}, \quad T^{a}=\frac{1}{2} \tau^{a}
$$

while $q^{A}$ and $\tilde{q}_{A}$ are quark hypermultiplets $(A=1,2)$. The covariant derivative in the adjoint representation is denoted by $D_{\mu}$. The coupling constants $g_{1}$ and $g_{2}$ correspond to the $U(1)$ and $S U(2)$ sectors, respectively. With our conventions, the $U(1)$ charges of the fundamental matter fields are $\pm 1 / 2$. The conventions in (21) are Euclidean. Moreover, $q$ and $\tilde{q}$ are the lowest (squark) components of the chiral superfields $Q$ and $\tilde{Q}$. Each flavor is composed of one $Q$ and one $\tilde{Q}$. The scalar complex field $a$ is an $\mathcal{N}=2$ superpartner of the gauge fields.

The potential $V\left(q^{A}, \tilde{q}_{A}, a^{a}, a\right)$ in the action 21$)$ is

$$
\begin{aligned}
& V\left(q^{A}, \tilde{q}_{A}, a^{a}, a\right)=\frac{g_{2}^{2}}{2}\left(\frac{i}{g_{2}^{2}} \varepsilon^{a b c} \bar{a}^{b} a^{c}+\bar{q}_{A} T^{a} q^{A}-\tilde{q}_{A} T^{a} \overline{\tilde{q}}^{A}\right)^{2} \\
& +\frac{g_{1}^{2}}{8}\left(\bar{q}_{A} q^{A}-\tilde{q}_{A} \overline{\tilde{q}}^{A}\right)^{2}+2 g_{2}^{2}\left|\tilde{q}_{A} T^{a} q^{A}\right|^{2}+\frac{g_{1}^{2}}{2}\left|\tilde{q}_{A} q^{A}-\xi\right|^{2} \\
& +\frac{1}{2} \sum_{A=1}^{N}\left\{\left|\left(a+2 T^{a} a^{a}\right) q^{A}\right|^{2}+\left|\left(a+2 T^{a} a^{a}\right) \overline{\tilde{q}}^{A}\right|^{2}\right\} .
\end{aligned}
$$

Here the sum over the repeated flavor indices $A$ is implied. In the simplest version the mass terms of the matter hypermultiplets are omitted. 
Let us discuss the vacuum structure of this model. We will limit ourselves to isolated vacua with the maximal possible value of condensed quarks - two. The vacua of the theory (21) are determined by the zeros of the potential (23). It is easy to see that the adjoint fields do not develop vacuum expectation values (VEVs),

$$
\langle\Phi\rangle=0
$$

where we defined the scalar adjoint matrix as

$$
\Phi=\frac{1}{2} a+T^{a} a^{a} .
$$

On the contrary, the squark fields do develop VEVs which have the color-flavor locked form (up to possible gauge transformations)

$$
\left\langle q^{k A}\right\rangle=\left\langle\overline{\tilde{q}}^{k A}\right\rangle=\sqrt{\frac{\xi}{2}}\left(\begin{array}{ll}
1 & 0 \\
0 & 1
\end{array}\right), \quad k=1,2, \quad A=1,2,
$$

where the squark fields is written as an $2 \times 2$ matrix in the color and flavor indices. The choice (26) makes the potential (23) vanish. For the time being the FayetIliopoulos parameter $\xi$ is assumed to be large, $\xi \gg \Lambda^{2}$.

The vacuum field (26) results in the spontaneous breaking of both gauge and flavor $S U(2)$ symmetries. A diagonal global $S U(2)$ survives, however,

$$
\mathrm{U}(2)_{\text {gauge }} \times \mathrm{SU}(2)_{\text {flavor }} \rightarrow \mathrm{SU}(2)_{C+F} .
$$

Thus, a color-flavor locking takes place in the vacuum. The above condensates imply that the basic theory under consideration is fully Higgsed and is at weak coupling provided $\xi$ is large.

\subsection{Non-Abelian Strings}

Why does the model described above support a novel type of strings, non-Abelian? The ANO string corresponds to a U(1) winding of the phase of the squark fields in the plane, perpendicular to the string axis,

$$
q^{k A} \underset{r \rightarrow \infty}{\longrightarrow} \sqrt{\frac{\xi}{2}} e^{i \alpha}\left(\begin{array}{ll}
1 & 0 \\
0 & 1
\end{array}\right),
$$

where $\alpha$ is the polar angle in the perpendicular plane. Its topological stability is due to $\pi_{1}(\mathrm{U}(1))=Z$. Now we have more options. It is well known that $\pi_{1}(S U(2))$ is 
trivial and, seemingly, there are no topologically stable strings other than those due to $\pi_{1}(U(1))$, i.e. the good old Abelian strings.

This is not the case, however. Observe that the center of the $\mathrm{SU}(2)$ group, $Z_{2}$, belongs to the $\mathrm{U}(1)$ factor too. This means that we need $\pi_{1}\left(S U(2) \times U(1) / Z_{2}\right)$ nontrivial. It is easy to see that $\pi_{1}\left(S U(2) \times U(1) / Z_{2}\right)=Z_{2}$. We can built a topologically stable $Z_{2}$ string.

One can split the $2 \pi$ windings in two halves: the first (from 1 to -1 ) is carried out in $U(1)$, while the second, from -1 to 1 in $S U(2)$ (e.g. around the third axis in the "isospace"). Correspondingly, the winding ansatz takes the form

$$
q^{k A} \longrightarrow \sqrt{\frac{\xi}{2}}\left(\begin{array}{cc}
e^{i \alpha} & 0 \\
0 & 1
\end{array}\right) \quad \text { or } \quad q^{k A} \longrightarrow \sqrt{\frac{\xi}{2}}\left(\begin{array}{cc}
1 & 0 \\
0 & e^{i \alpha}
\end{array}\right)
$$

depending on which of the two combination of generators $T_{\mathrm{U}(1)} \pm T_{\mathrm{SU}(2)}^{3}$ we use.

It is clear, that the ansatz (29) breaks the color-flavor locked SU(2) down to U(1). The particular way of embedding is unimportant. Instead of $T^{3}$ we could have chosen any other generator of $S U(2)$. In other words, the existence of the string based on (29) implies the existence of the whole family of strings parametrized by the coset $S U(2) / U(1)$ moduli. Then, the theory of the moduli fields on the string world sheet is obviously the $C P(1)$ model. It is asymptotically free in the ultroviolet (UV) and strongly coupled in the infrared (IR). Since the string is $1 / 2$ BPS saturated, the world-sheet model has four supercharges. Thus, we arrive at the $\mathcal{N}=(2,2)$ model. The existence of the orientational moduli implies that the flux through the string does not have a preferred orientation inside $S U(2)$. This string is genuinely non-Abelian.

If the tension of the ANO string is $4 \pi \xi$, the tension of the non-Abelian string is $2 \pi \xi$. For arbitrary $N$ the tension of the ANO string is $2 N \pi \xi$, while the tension of our non-Abelian string remains $2 \pi \xi$; it is $N$-independent. This is an important consequence of the BPS saturation.

\subsection{Kinks as Confined Monopoles}

There are two degenerate vacua in $\mathcal{N}=2 C P(1)$ model. This is dictated by the Witten index. The existence of two isolated vacua are not seen classically, since classically we do not see mass gap generation. They are labeled by the fermion condensate $\langle\bar{\psi} \psi\rangle$, which can take two values, in much the same way as the gluino condensate in $\mathcal{N}=1 S U(2)$ Yang-Mills theory.

From the bulk standpoint this means that there exist two distinct strings, both with the tension value $2 \pi \xi$. If so, there should exist a junction of these two strings. 
Returning to two dimensions we can say that this junction is a kink interpolating between two distinct vacua of the supersymmetric $C P(1)$ model (Fig. 6).

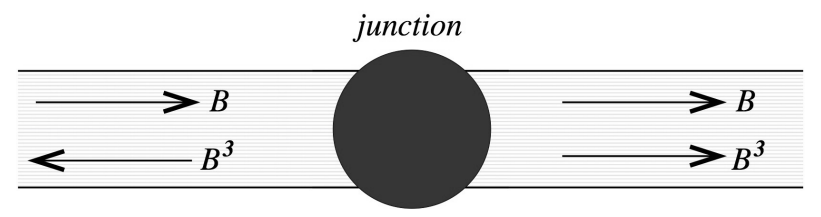

Figure 6: $C P(1)$ model kink as a junction of two degenerate strings.

The kink itself is BPS saturated since, as I mentioned above, $\mathcal{N}=(2,2)$ algebra in $C P(1)$ has a central extension. Therefore, the mass of the kink is exactly calculable.

Moreover, using supersymmetry one can prove that this two-dimensional kink is a reincarnation of a confined monopole, i.e. a monopole with strings attached to it. To this end we must use exact holomorphic dependences protected by supersymmetry.
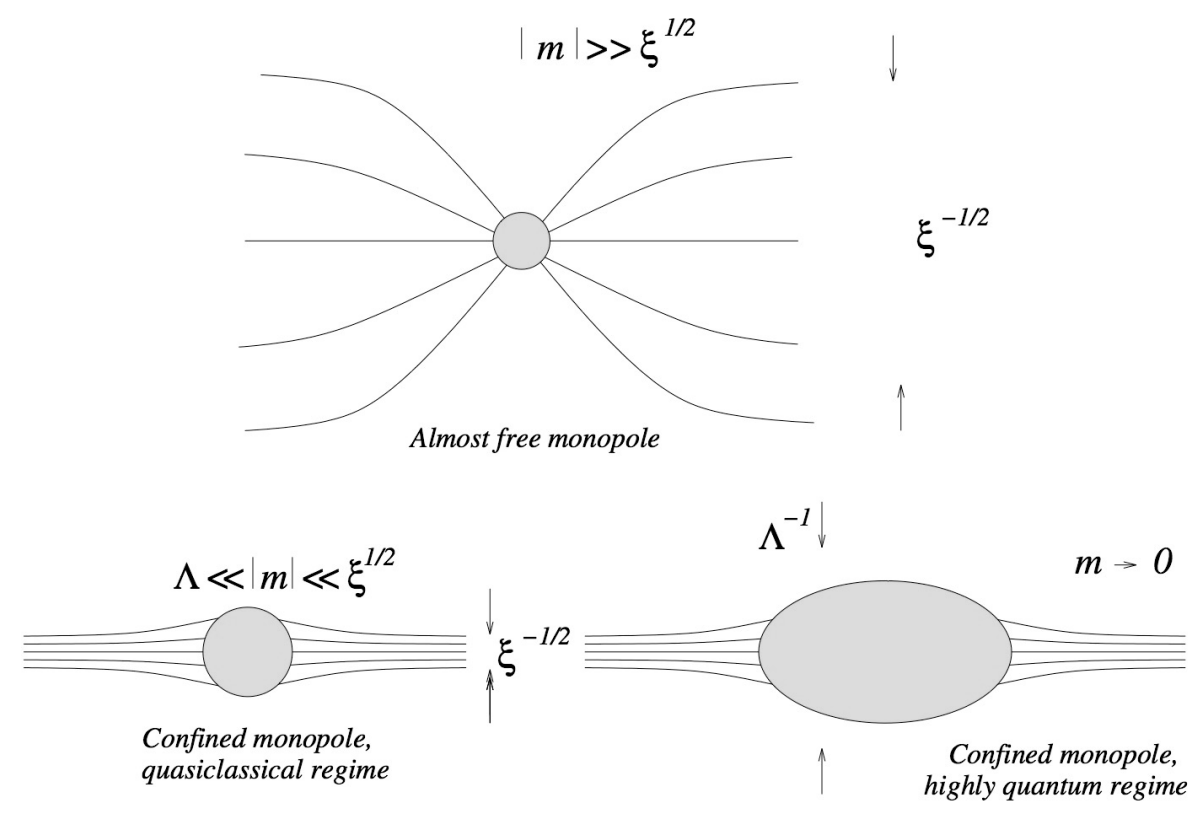

Figure 7: Evolution of the confined monopoles.

Since so far we set the quark mass term $m=0$, the theory under consideration has two parameters of dimension of mass: the string tension $\xi$ and the dynamical scale $\Lambda$. Note a "miraculous" fact that $\Lambda$ is one and the same in the bulk theory and in the string world-sheet model! In the limit $m_{1}=m_{2}=0$ the kink is unrecognizable 
as a $4 \mathrm{D}$ monopole. However, if we introduce another holomorphic parameter of dimension of mass (the so-called twisted mass which preserves $\mathcal{N}=2$ ) and allow it to vary continuously, we will see that starting from the classical 't Hooft-Polyakov monopole, and analytically continue (smoothly, with no singularities) this parameter, we arrive at the kink on the string.

The crucial parameter is the squark mass difference $\Delta m_{12}=m_{1}-m_{2} \equiv 2 m \neq 0$. It is clear that $\Delta m_{12}$ is a complex parameter, and so is $\Lambda$. At the same time $\xi$ is a real parameter, and, as a result, the appropriate central charges cannot depend on $\xi$. At $\xi=0$ and $\Delta m_{12} \neq 0$ we are on the Coulomb branch.

If $|m| \gg \xi$ (or, alternatively, $\xi \rightarrow 0$ ), we arrive (in the bosonic sector) at the Georgi-Glashow model, with the classical 't Hooft-Polyakov monopoles. Making $\xi$ nonvanishing but small we attach strings to the 't Hooft-Polyakov monopole, although these flux tubes form far away from its core, which (the core) remains easily recognizable. Evolving towards smaller values of $|m|$ and larger values of $\xi$ we arrive, at the end of the evolution process, at the kink described above. Thus, indeed, this kink is the monopole's apparent heir. Figure 7 illustrates this analytic continuation.

Four-dimensional expression for the free monopole mass (i.e. on the Coulomb branch at $\xi=0$ ) obtained in [8] in our problem reduces to

$$
M_{\mathrm{mon}}^{\text {Coulomb }}=\sqrt{2}\left|a_{D}^{3}\left(a^{3}=-\frac{\Delta m_{12}}{\sqrt{2}}\right)\right|
$$

where $a_{D}^{3}$ is the dual Seiberg-Witten potential for the $S U(2)$ gauge group. Now, if one switches on a Fayet-Iliopoulos parameter $\xi \neq 0$, a priori one could expect corrections to the monopole mass depending on $\sqrt{\xi} / \Lambda$ but in fact they are forbidden by $\mathrm{U}(1)_{R}$ charges. Therefore,

$$
M_{\mathrm{mon}}^{\text {Coulomb }}=M_{\mathrm{mon}}^{\text {confinement }} .
$$

On the other hand, the monopole mass in the confinement phase is given by the kink mass in the $C P(1)$ model,

$$
M_{\mathrm{kink}}=\left|Z_{\mathrm{kink}}\right|
$$

where $Z_{\text {kink }}$ is given by the following formula:

$$
\langle Z\rangle_{\mathrm{kink}}=-\frac{i}{2 \pi}\left\{\Delta m_{12} \log \frac{\Delta m_{12}+\sqrt{\Delta m_{12}^{2}+4 \Lambda^{2}}}{\Delta m_{12}-\sqrt{\Delta m_{12}^{2}+4 \Lambda^{2}}}-2 \sqrt{\Delta m_{12}^{2}+4 \Lambda^{2}}\right\} .
$$

If the two-dimensional kink indeed represents a confined four-dimensional monopole, then Eq. (32) can be extended,

$$
M_{\text {mon }}^{\text {Coulomb }} \leftrightarrow M_{\text {mon }}^{\text {confinement }} \leftrightarrow M_{\text {kink }} \cdot
$$


Comparing (30) and (33) we confirm exact agreement and thus establish the 2D$4 \mathrm{D}$ correspondence. This and other cases of the 2D-4D correspondence are reviewed in 43,52 .

A remarkably close parallel between four-dimensional $S U(2)$ Yang-Mills theory with $N_{f}=2$ on the Coulomb branch and the two-dimensional $C P(1)$ model was noted in [53]. The coincidence observed by Dorey remained mysterious and could not be understood before the advent of non-Abelian strings.

\section{4 $\mathcal{N}=1$ bulk theories give rise to $\mathcal{N}=(0,2)$ sigma models on the world sheet}

If we deform the basic model described in Sec. 4.1 by adding $\mu \operatorname{Tr}\left(a^{2}\right)$ term in the superpotential (this term breaks the bulk symmetry down to $\mathcal{N}=1$ ) then a nonminimal heterotic $C P(N-1)$ model emerges on the string world sheet [54,55. This is a remarkably rich two-dimensional model. Supersymmetry on the world sheet is spontaneously broken [56] in this model. At the same time there are still $N$ degenerate vacua and, therefore, the kinks representing confined monopoles survive. The world-sheet dynamics becomes highly nontrivial if we add twisted masses. In this case the large- $N$ solution exhibits three distinct phases [56] depending on the ratio $m / \Lambda$.

\section{A non-Abelian string can be critical}

Most of the non-Abelian strings we constructed and explored are not ultravioletcomplete in the sense of Polchinski and Strominger [57]. This means that they cannot be quantized as strings at energies higher than the inverse thickness of the vortex string at hand. It is clear that at such energies we have to take into account higher derivative corrections. The blow up of higher derivative terms in the worldsheet theory reflects a finite thickness of the solitonic vortex strings. Some time ago a question was raised [58] (see also [59]) whether one can find a solitonic string which is critical. To this end it must be a ten-dimensional superstring, of course.

If such solitonic string exists it must satisfy a number of conditions, namely,

(i) it should be infinitely thin, implying that higher derivative terms on the world sheet can be ignored;

(ii) The world-sheet theory must be conformally invariant;

(iii) The theory must have the critical value of the Virasoro central charge.

The conditions (i), (ii) and (iii) above are met by the non-Abelian vortex string 
[51] supported in four-dimensional $\mathcal{N}=2$ supersymmetric QCD with the $\mathrm{U}(N)$ gauge group, $N_{f}=2 N$ matter hypermultiplets and the Fayet-Iliopoulos parameter $\xi$. To ensure the appropriate value of the Virasoro central charge and make the four-dimensional problem effectivly ten-dimensional we need $N=2$ and $N_{f}=4$.

The non-Abelian vortex string meeting the above conditions has $\mathcal{N}=(2,2)$ supersymmetry on its world sheet. In contradistinction to the $N=N_{f}$ case discussed in Sect. 4 it has not only translational and orientational moduli, but the so-called size moduli as well [60]. This is due to the fact that the dynamics of orientational and size moduli are described by two-dimensional sigma model known in physics as $W C P\left(N, N_{f}-N\right)$ model $\left.\right|^{8}$ and in mathematics as

$$
\mathcal{O}(-1)_{\mathbb{C P}^{1}}^{\oplus\left(N_{f}-N\right)} .
$$

For $N_{f}=2 N$ the model is conformal and the condition (ii) above is satisfied. Moreover for $N=2$ the dimension of orientational/size moduli space is six and they can be combined with four translational moduli to form a ten-dimensional space required for critical superstrings..$^{9}$ Thus the condition (iii) is satisfied too [58]. For $N=2$ the sigma model target space is a six-dimensional non-compact Calabi-Yau manifold $Y_{6}$, namely, the resolved conifold.

Given that the conditions (ii) and (iii) are met, we assumed [58] that vortex string satisfies the thin-string condition (i) at strong coupling,

$$
\ell \rightarrow 0, \text { at } g^{2} \rightarrow g_{c}^{2} \sim 1,
$$

where $\ell$ is the string thickness.

As well known 62, 63, the bulk theory at hand possesses a strong-weak coupling duality ${ }^{10}$ So does the two-dimensional world sheet theory: the $\operatorname{WCP}\left(N, N_{f}-N\right)$ model is self-dual under the reflection of the coupling constant $\beta$,

$$
\beta \rightarrow \beta_{D}=-\beta .
$$

Under transformation (37) the orientational and size moduli interchange. Note that the $2 \mathrm{D}$ coupling constant $\beta$ can be complexified by including the $\theta$ term in the action

\footnotetext{
${ }^{8}$ Both the orientational and the size moduli have logarithmically divergent norms, see e.g. 60 . After an appropriate infrared regularization, logarithmically divergent norms can be absorbed into the definition of relevant two-dimensional fields. In fact, the world-sheet theory on the semilocal non-Abelian string is not exactly the $\operatorname{WCP}\left(N, N_{F}-N\right)$ model [61], there are minor differences inessential in the infrared.

${ }^{9}$ It corresponds to $\widehat{c}=\frac{c}{3}=3$.

${ }^{10}$ Argyres et al. proved this duality for $\xi=0$. It should allow one to study the bulk theory at strong coupling in terms of weakly coupled dual theory at $\xi \neq 0$ too.
} 
of the $C P(N-1)$ model,

$$
\beta \rightarrow \beta+i \frac{\theta_{2 D}}{2 \pi}
$$

As a result, one can derive an exact 2D-4D map between the coupling constants, see 64 for a detailed analysis. The $4 \mathrm{D}$ self-dual point $g^{2}=4 \pi$ is mapped onto the $2 \mathrm{D}$ self-dual point $\beta=0$.

The thin string hypothesis is equivalent to the following statement

$$
\ell^{-2} \rightarrow \xi \times\left\{\begin{array}{cc}
g^{2}, & g^{2} \ll 1 \\
\infty, & g^{2} \rightarrow 4 \pi \\
16 \pi^{2} / g^{2}, & g^{2} \gg 1
\end{array}\right.
$$

where the dependence of $\ell^{-2}$ at small and large $g^{2}$ follows from the weak coupling formula for the Higgsed bulk gauge bosons and duality [62,63]. In terms of $\beta$ the critical point is $\beta=0$. At this point the target space of the $W C P(2,2)$ part of the world-sheet model develops a conical singularity. The number of real (bosonic) degrees of freedom parametrizing $\operatorname{WCP}(2,2)$ is six. Adding four translational moduli we get ten-dimensional space The critical string we arrived at can be viewed as a type IIA superstring, a version of the Kutasov-Vafa little string. The target space is $\mathbb{R}^{4} \times W C P(2,2)=\mathbb{R}^{4} \times Y_{6}$ where $Y_{6}$ is a non-compact Calabi-Yau conifold.

\section{Briefly about spectrum}

\subsection{Massless states}

First, we addressed massless four-dimensional excitations of the quantized string. To this end zero modes of appropriate operators in the $Y_{6}$ background were found. At first sight one might think that there are no normalizable zero modes at all, because our our Calabi-Yau space is non-compact. As a matter of fact, at the selfdual value of $\beta=0$ a marginally normalizable scalar zero mode exists!

Our analysis led us to the conclusion that the only road leading to the above zero mode is as follows:

$$
\delta G_{i j}=\phi_{4}(x) \delta g_{i j}(y)
$$

where $x_{\mu}$ and $y_{i}$ are the coordinates on $\mathbb{R}^{4}$ and $Y_{6}$, respectively, and $G_{i j}$ is the metric on $Y_{6}$. Then we studied the relevant Lichnerowicz equation on $Y_{6}$ [58, 59]. Solutions of this equation for the Calabi-Yau spaces reduce to deformations of the Kähler form or deformations of complex structure [65,66]. In the former case we deal with the resolved conifold while in the latter case with the deformed conifold. The 
difference between the two lies in the method of smoothing the conifold singularity. For the resolved conifold one introduces a non-zero (but small) $\beta$ preserving the Kähler structure and Ricci-flatness of the metric. The explicit metric for the resolved conifold can be found in $67-69]$. The resolved conifold has no normalizable zero modes. In particular, it is demonstrated in [59] that the four-dimensional scalar $\beta$ associated with the Kähler form deformation is non-normalizable.

At the same time deformation of the complex structure [65] does lead to a marginally normalizable four-dimensional scalar localized on the string in the same sense as the orientational and size zero modes are localized on the vortex-string solution.

Thus, a four-dimensional massless scalar $b$ is a part of a four-dimensional $\mathcal{N}=2$ hypermultiplet. This implies that we observe a new Higgs branch in the bulk which is developed only at the self-dual value of the bulk coupling constant $g^{2}=4 \pi$.

\subsection{Massive states}

The critical string theory on the conifold is hard to use for calculating the spectrum of massive string modes because (unlike Sect. (6.1)) the supergravity approximation does not work. However, in the given problem one can act in a different way and use the equivalent formulation of the theory as a non-critical $c=1$ string theory with the Liouville field and a compact scalar at the self-dual radius [70,71]. Non-critical $c=1$ string theory is formulated on the target space

$$
\mathbb{R}^{4} \times \mathbb{R}_{\phi} \times S^{1},
$$

where $\mathbb{R}_{\phi}$ is a real line associated with Liouville field $\phi$.

Our first results on the spectrum of the solitonic-critical string [72 are presented in Fig. 8 which shows the masses of $4 \mathrm{D}$ spin- 0 and spin- 2 states as a function of the baryonic charge. The results of the current studies, including the 4D hypermultiplet structure, will be reported elsewhere [73].

\section{Instead of conclusions. A digression}

I will not summarize my talk for many reasons. My presentation is rather concise (although covers many topics on which I worked since 1980) and hopefully accessible to the interested reader, being supplemented by a detailed list of references below. Instead, I'd like to discuss a rather pessimistic question that was posed by Richard Feynman [74 fifty years ago. Feynman wrote: 


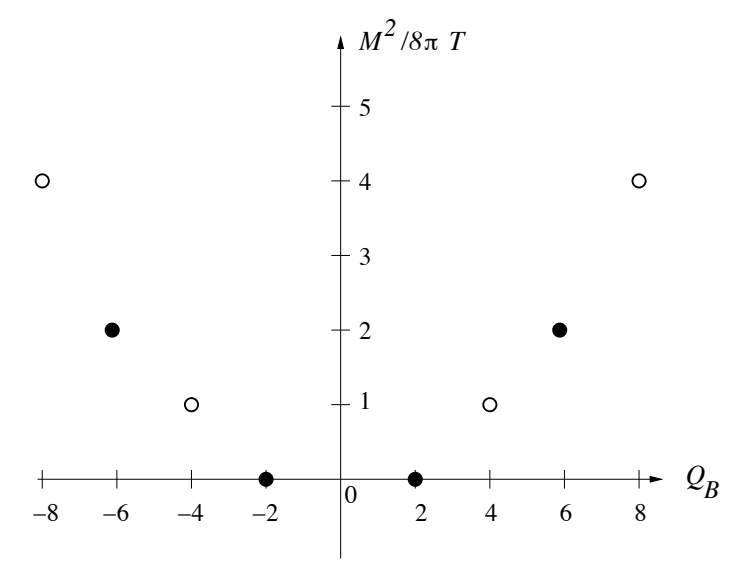

Figure 8: Spectrum of spin-0 and spin-2 states as a function of the baryonic charge. Closed and open circles denote spin-0 and spin-2 states, respectively.

What will happen [to our science] ultimately? We are going along guessing the laws; how many laws are we going to have to guess? I do not know. Some of my colleagues say that this fundamental aspect of our science will go on; but I think there will certainly not be perpetual novelty, say for a thousand years. This thing cannot keep on going so that we are always going to discover more and more new laws. If we do, it will become boring that there are so many levels one underneath the other. It seems to me that what can happen in the future is either that all the laws become known - that is, if you had enough laws you could compute consequences and they would always agree with experiment, which would be the end of the line - or it may happen that the experiments get harder and harder to make, more and more expensive, so you get 99.9 per cent of the phenomena, but there is always some phenomenon which has just been discovered, which is very hard to measure, and which disagrees; and as soon as you have the explanation of that one there is always another one, and it gets slower and slower and more and more uninteresting. That is another way it may end. But I think it has to end in one way or another.

Any thoughts? 


\section{Acknowledgments}

I am grateful to Alexei Yung for endless discussions. Correspondence with E. Gerchkovitz, A. Karasik and A. Kataev is acknowledged. This work is supported in part by DOE grant de-sc0011842.

\section{References}

[1] Extension of the Algebra of Poincare Group Generators and Violation of P Invariance, JETP Lett. 13, 323 (1971), [reprinted in Supersymmetry, Ed. S. Ferrara, (NorthHollands/World Sci, 1987), vol. 1, page 7].

[2] W. Pauli, Pauli Lectures on Physics, Vol. 6, Selected Topics on Field Quantization, (MIT Press, Cambridge, MA, 1973), p. 33.

[3] E. Likhtman, FIAN Report No. 41 (1971), [Reprinted in English in E. Likhtman, About SuSy 1970, in Proceedings of the Intern. Symp. "Celebrating 30 Years of Supersymmetry," Eds. K. Olive, S. Rudaz and M. Shifman (Nucl. Phys. B (Proc. Suppl.) 101 (2001)), pp. 5-14].

[4] V. A. Novikov, M. A. Shifman, A. I. Vainshtein and V. I. Zakharov, Exact Gell-MannLow Function of Supersymmetric Yang-Mills Theories from Instanton Calculus, Nucl. Phys. B 229, 381 (1983).

[5] A. A. Belavin, A. M. Polyakov, A. S. Schwartz and Y. S. Tyupkin, Pseudoparticle Solutions of the Yang-Mills Equations, Phys. Lett. 59B, 85 (1975).

[6] V. A. Novikov, M. A. Shifman, A. I. Vainshtein and V. I. Zakharov, Supersymmetric Instanton Calculus (Gauge Theories with Matter), Nucl. Phys. B 260, 157 (1985)

[7] M. A. Shifman, Exact results in gauge theories: Putting supersymmetry to work. The 1999 Sakurai Prize Lecture, Int. J. Mod. Phys. A 14, 5017 (1999) hep-th/9906049].

[8] N. Seiberg and E. Witten, Electric-magnetic duality, monopole condensation, and confinement in $\mathcal{N}=2$ supersymmetric Yang-Mills theory, Nucl. Phys. B 426, 19 (1994) Erratum: [Nucl. Phys. B 430, 485 (1994)] hep-th/9407087]; Monopoles, duality and chiral symmetry breaking in $\mathcal{N}=2$ supersymmetric $Q C D$, Nucl. Phys. B 431, 484 (1994) [hep-th/9408099]. 
[9] V. A. Novikov, M. A. Shifman, A. I. Vainshtein and V. I. Zakharov, Beta Function in Supersymmetric Gauge Theories: Instantons Versus Traditional Approach, Phys. Lett. 166B, 329 (1986).

[10] M. A. Shifman and A. I. Vainshtein, Solution of the Anomaly Puzzle in SUSY Gauge Theories and the Wilson Operator Expansion, Nucl. Phys. B 277, 456 (1986).

[11] V. Y. Shakhmanov and K. V. Stepanyantz, Three-loop NSVZ relation for terms quartic in the Yukawa couplings with the higher covariant derivative regularization, Nucl. Phys. B 920, 345 (2017) [arXiv:1703.10569 [hep-th]]; A. E. Kazantsev, V. Y. Shakhmanov and K. V. Stepanyantz, New form of the exact NSVZ $\beta$-function: the three-loop verification for terms containing Yukawa couplings, arXiv:1803.06612 [hep-th].

[12] Z. Komargodski and N. Seiberg, Comments on the Fayet-Iliopoulos Term in Field Theory and Supergravity, JHEP 0906, 007 (2009) arXiv:0904.1159 [hep-th]]; Comments on Supercurrent Multiplets, Supersymmetric Field Theories and Supergravity, JHEP 1007, 017 (2010); arXiv:1002.2228 [hep-th]]. T. T. Dumitrescu and N. Seiberg, Supercurrents and Brane Currents in Diverse Dimensions, JHEP 1107, 095 (2011) arXiv:1106.0031 [hep-th]].

[13] N. Seiberg, Electric - magnetic duality in supersymmetric non-Abelian gauge theories, Nucl. Phys. B 435, 129 (1995); hep-th/9411149]. see also K. A. Intriligator and N. Seiberg, Lectures on supersymmetric gauge theories and electric-magnetic duality, Nucl. Phys. Proc. Suppl. 45BC, 1 (1996) hep-th/9509066.

[14] V. A. Novikov, M. A. Shifman, A. I. Vainshtein and V. I. Zakharov, Instantons And Exact Gell-Mann-Low Function Of Supersymmetric O(3) Sigma Model, Phys. Lett. 139B, 389 (1984); A. Y. Morozov, A. M. Perelomov and M. A. Shifman, Exact GellMann-Low Function Of Supersymmetric Kähler Sigma Models, Nucl. Phys. B 248, 279 (1984).

[15] G. W. Moore and P. C. Nelson, The Etiology of $\sigma$ Model Anomalies, Commun. Math. Phys. 100, 83 (1985).

[16] J. Chen, X. Cui, M. Shifman and A. Vainshtein, On isometry anomalies in minimal $\mathcal{N}=(0,1)$ and $\mathcal{N}=(0,2)$ sigma models, Int. J. Mod. Phys. A 31, no. 27, 1650147 (2016) arXiv:1510.04324 [hep-th]]; Anomalies of minimal $\mathcal{N}=(0,1)$ and $\mathcal{N}=(0,2)$ sigma models on homogeneous spaces, J. Phys. A 50, no. 2, 025401 (2017) arXiv:1511.08276 [hep-th]].

[17] X. Cui and M. Shifman, N=(0,2) Deformation of CP(1) Model: Two-dimensional Analog of N=1 Yang-Mills Theory in Four Dimensions, Phys. Rev. D 85, 045004 (2012) arXiv:1111.6350 [hep-th]]. 
[18] T. Banks and A. Zaks, On the Phase Structure of Vector-Like Gauge Theories with Massless Fermions, Nucl. Phys. B 196, 189 (1982).

[19] J. Chen, X. Cui, M. Shifman and A. Vainshtein, $N=(0,2)$ deformation of (2, 2) sigma models: Geometric structure, holomorphic anomaly, and exact $\beta$ functions, Phys. Rev. D 90, no. 4, 045014 (2014) arXiv:1404.4689 [hep-th]].

[20] M. Edalati and D. Tong, Heterotic Vortex Strings, JHEP 0705, 005 (2007) hepth/0703045 [HEP-TH]]; M. Shifman and A. Yung, Heterotic Flux Tubes in $\bar{N}=$ $2 S Q C D$ with $N=1$ Preserving Deformations, Phys. Rev. D 77, 125016 (2008) [Erratum-ibid. D 79, 049901 (2009)] [arXiv:0803.0158 [hep-th]]; M. Shifman and A. Yung, Two-Dimensional Sigma Models Related to Non-Abelian Strings in Super-YangMills, arXiv:1401.7067 [published in Pomeranchuk 100, Eds. A. Gorsky and M. Vysotsky, (World Scientific, Singapore, 2014), p. 181].

[21] M. Shifman and K. Stepanyantz, Exact Adler Function in Supersymmetric QCD, Phys. Rev. Lett. 114, no. 5, 051601 (2015) |arXiv:1412.3382 [hep-th]]; Derivation of the exact expression for the D function in $N=1 S Q C D$, Phys. Rev. D 91, 105008 (2015) arXiv:1502.06655 [hep-th]].

[22] A. L. Kataev, A. E. Kazantsev and K. V. Stepanyantz, The Adler D function for $\mathcal{N}=1 S Q C D$ Regularized by Higher Covariant Derivatives in the Three-Loop Approximation, Nucl. Phys. B 926 (2018) 295 [arXiv:1710.03941 [hep-th]].

[23] A. L. Kataev and K. V. Stepanyantz, NSVZ Scheme with the Higher Derivative Regularization for $\mathcal{N}=1$ SQED, Nucl. Phys. B 875 (2013) 459 [arXiv:1305.7094 [hep-th]].

[24] V. A. Novikov, M. A. Shifman, A. I. Vainshtein and V. I. Zakharov, Instanton Effects in Supersymmetric Theories, Nucl. Phys. B 229, 407 (1983).

[25] V. A. Novikov, M. A. Shifman, A. I. Vainshtein and V. I. Zakharov, Supersymmetric Instanton Calculus (Gauge Theories with Matter), Nucl. Phys. B 260, 157 (1985);

[26] M. A. Shifman and A. I. Vainshtein, On Gluino Condensation in Supersymmetric Gauge Theories. SU(N) and $O(N)$ Groups, Nucl. Phys. B 296, 445 (1988); A. Y. Morozov, M. A. Olshanetsky and M. A. Shifman, Gluino Condensate in Supersymmetric Gluodynamics, Nucl. Phys. B 304, 291 (1988).

[27] N. M. Davies, T. J. Hollowood, V. V. Khoze and M. P. Mattis, Gluino condensate and magnetic monopoles in supersymmetric gluodynamics, Nucl. Phys. B 559, 123 (1999) hep-th/9905015].

[28] N. A. Nekrasov, Seiberg-Witten prepotential from instanton counting, Adv. Theor. Math. Phys. 7, no. 5, 831 (2003) [hep-th/0206161]. 
[29] J. T. Łopuszański and M. Sohnius, Karlsruhe Report Print-74-1269 (unpublished).

[30] R. Haag, J. T. Łopuszański and M. Sohnius, Nucl. Phys. B 88, 257 (1975) [Reprinted in Supersymmetry, Ed. S. Ferrara, (North-Holland/World Scientific, 1987) Vol. 1, p. 51].

[31] E. Witten and D. I. Olive, Supersymmetry Algebras That Include Topological Charges, Phys. Lett. 78B, 97 (1978).

[32] M. A. Shifman, A. I. Vainshtein and M. B. Voloshin, Anomaly and quantum corrections to solitons in two-dimensional theories with minimal supersymmetry, Phys. Rev. D 59, 045016 (1999) hep-th/9810068.

[33] A. Losev, M. A. Shifman and A. I. Vainshtein, Counting supershort supermultiplets, Phys. Lett. B 522, 327 (2001) hep-th/0108153]; Single state supermultiplet in (1+1)dimensions, New J. Phys. 4, 21 (2002) [hep-th/0011027].

[34] G. R. Dvali and M. A. Shifman, Phys. Lett. B 396, 64 (1997) (E) B 407, 452 (1997) hep-th/9612128.

[35] E. Witten, Branes and the dynamics of QCD, Nucl. Phys. B 507, 658 (1997) hepth/9706109.

[36] B. S. Acharya and C. Vafa, On domain walls of $N=1$ supersymmetric Yang-Mills in four-dimensions, hep-th/0103011.

[37] A. Kovner, M. A. Shifman and A. V. Smilga, Domain walls in supersymmetric YangMills theories, Phys. Rev. D 56, 7978 (1997) doi:10.1103/PhysRevD.56.7978 hepth/9706089.

[38] A. Ritz, M. Shifman and A. Vainshtein, Counting domain walls in $N=1$ superYangMills, Phys. Rev. D 66, 065015 (2002) hep-th/0205083;

[39] A. Ritz, M. Shifman and A. Vainshtein, Enhanced worldvolume supersymmetry and intersecting domain walls in N=1 SQCD, Phys. Rev. D 70, 095003 (2004) hepth/0405175.

[40] A. Ritz and A. Shukla, Domain wall moduli in softly broken $S Q C D$ at $\theta=\pi$, to appear.

[41] D. Gaiotto, A. Kapustin, Z. Komargodski and N. Seiberg, Theta, Time Reversal, and Temperature, JHEP 1705, 091 (2017) arXiv:1703.00501 [hep-th]]. 
[42] A. Losev and M. Shifman, N=2 sigma model with twisted mass and superpotential: Central charges and solitons, Phys. Rev. D 68, 045006 (2003) hep-th/0304003 (Section 10); M. Shifman, A. Vainshtein and R. Zwicky, Central charge anomalies in 2-D sigma models with twisted mass, J. Phys. A 39, 13005 (2006) hep-th/0602004.

[43] M. Shifman and A. Yung, Supersymmetric Solitons, (Cambridge University Press, 2009).

[44] M. Shifman and A. Yung, Two-Dimensional Sigma Models Related to Non-Abelian Strings in Super-Yang-Mills, arXiv:1401.7067 [published in Pomeranchuk 100, Eds. A. Gorsky and M. Vysotsky, (World Scientific, Singapore, 2014), p. 181].

[45] From superYang-Mills theory to QCD: Planar equivalence and its implications, hepth/0403071, published in From Fields to Strings: Circumnavigating Theoretical Physics, Eds. M. Shifman, A. Vainshtein and J. Wheater (World Scientific, Singapore, 2005), Vol. 1, page 353.

[46] A. Armoni, M. Shifman and G. Veneziano, Exact results in non-supersymmetric large $N$ orientifold field theories, Nucl. Phys. B 667, 170 (2003) hep-th/0302163; Refining the proof of planar equivalence, Phys. Rev. D 71, 045015 (2005) hep-th/0412203.

[47] A. Armoni, M. Shifman and G. Veneziano, QCD quark condensate from SUSY and the orientifold large $N$ expansion, Phys. Lett. B 579, 384 (2004) hep-th/0309013; A. Armoni, M. Shifman, G. Shore and G. Veneziano, The quark condensate in multiflavour QCD: planar equivalence confronting lattice simulations, Phys. Lett. B 741, 184 (2015) arXiv:1412.3389 [hep-th]].

[48] All you need is N: Baryon spectroscopy in two large $N$ limits, Phys. Rev. D 80, 036002 (2009) |arXiv:0906.2400 [hep-ph]]; M. I. Buchoff, A. Cherman and T. D. Cohen, Color Superconductivity at Large N: A New Hope, Phys. Rev. D 81, 125021 (2010) arXiv:0910.0470 [hep-ph]]; F. Buisseret and C. Semay, Phys. Rev. D 82, 056008 (2010) [arXiv:1006.4729 [hep-ph]]; T. D. Cohen, Nuclear and hadronic physics at large $N_{c}$, PoS QNP 2012, 022 (2012).

[49] T. D. Cohen and R. F. Lebed, Tetraquarks with exotic flavor quantum numbers at large $N_{c}$ in $Q C D(A S)$, Phys. Rev. D 89, no. 5, 054018 (2014) arXiv:1401.1815 [hep-ph]].

[50] A. A. Abrikosov, On the Magnetic properties of superconductors of the second kind," Sov. Phys. JETP 5, 1174 (1957); H. B. Nielsen and P. Olesen, Vortex Line Models for Dual Strings, Nucl. Phys. B 61, 45 (1973).

[51] A. Hanany and D. Tong, Vortices, instantons and branes, JHEP 0307, 037 (2003) [hep-th/0306150]; Vortex strings and four-dimensional gauge dynamics, JHEP 0404, 
066 (2004) hep-th/0403158]; R. Auzzi, S. Bolognesi, J. Evslin, K. Konishi and A. Yung, Non-Abelian superconductors: Vortices and confinement in N=2 SQCD, Nucl. Phys. B 673, 187 (2003) hep-th/0307287]; M. Shifman and A. Yung, NonAbelian string junctions as confined monopoles, Phys. Rev. D 70, 045004 (2004) hepth/0403149.

[52] M. Shifman and A. Yung, Quantum Deformation of the Effective Theory on NonAbelian string and 2D-4D correspondence, Phys. Rev. D 89, no. 6, 065035 (2014) arXiv:1401.1455 [hep-th]].

[53] N. Dorey, The BPS spectra of two-dimensional supersymmetric gauge theories with twisted mass terms, JHEP 9811, 005 (1998) hep-th/9806056.

[54] M. Edalati and D. Tong, Heterotic Vortex Strings, JHEP 0705, 005 (2007) hepth/0703045 [HEP-TH]].

[55] M. Shifman and A. Yung, Heterotic Flux Tubes in $N=2$ SQCD with $N=1$ Preserving Deformations, Phys. Rev. D 77, 125016 (2008) Erratum: [Phys. Rev. D 79, 049901 (2009)] arXiv:0803.0158 [hep-th]].

[56] M. Shifman and A. Yung, Large- $N$ Solution of the Heterotic N=(0,2) TwoDimensional CP(N-1) Model, Phys. Rev. D 77, 125017 (2008), Erratum: [Phys. Rev. D 81, 089906 (2010)]; arXiv:0803.0698 [hep-th]]; P. A. Bolokhov, M. Shifman and A. Yung, Large- $N$ Solution of the Heterotic $C P(N-1)$ Model with Twisted Masses, Phys. Rev. D 82, no. 2, 025011 (2010) Erratum: [Phys. Rev. D 89, no. 2, 029904 (2014)] [arXiv:1001.1757 [hep-th]].

[57] J. Polchinski and A. Strominger, Effective string theory, Phys. Rev. Lett. 67, 1681 (1991).

[58] M. Shifman and A. Yung, Critical String from Non-Abelian Vortex in Four Dimensions, Phys. Lett. B 750, 416 (2015) arXiv:1502.00683 [hep-th]].

[59] P. Koroteev, M. Shifman and A. Yung, Studying Critical String Emerging from NonAbelian Vortex in Four Dimensions, Phys. Lett. B 759, 154 (2016) arXiv:1605.01472 [hep-th]]; Non-Abelian Vortex in Four Dimensions as a Critical String on a Conifold, Phys. Rev. D 94, no. 6, 065002 (2016) arXiv:1605.08433 [hep-th]]; Non-Abelian Vortex in Four Dimensions as a Critical Superstring, JETP Letters, 105, no.1, 60 arXiv:1611.03111 [hep-th].

[60] M. Shifman and A. Yung, Non-Abelian semilocal strings in N=2 supersymmetric $Q C D$, Phys. Rev. D 73, 125012 (2006) [hep-th/0603134]. 
[61] M. Shifman, W. Vinci and A. Yung, Effective World-Sheet Theory for Non-Abelian Semilocal Strings in $N=2$ Supersymmetric QCD, Phys. Rev. D 83, 125017 (2011) arXiv:1104.2077 [hep-th]].

[62] P. Argyres, M. R. Plesser and A. Shapere, The Coulomb Phase of N=2 Supersymmetric $Q C D$ Phys. Rev. Lett. 75, 1699 (1995). hep-th/9505100.

[63] P. Argyres, M. Plesser and N. Seiberg, The Moduli Space of $\mathcal{N}=2$ SUSY QCD and Duality in $\mathcal{N}=1$ SUSY QCD, Nucl. Phys. B471, 159 (1996).

[64] E. Gerchkovitz and A. Karasik, New Vortex-String Worldsheet Theories from Supersymmetric Localization, arXiv:1711.03561 [hep-th].

[65] A. Neitzke and C. Vafa, Topological strings and their physical applications, arXiv:hepth/0410178.

[66] S. Gukov, C. Vafa and E. Witten CFT's from Calabi-Yau four folds, Nucl. Phys. B584, 69 (2000) arXiv:hep-th/0410178.

[67] P. Candelas and X. C. de la Ossa, Comments on conifolds, Nucl. Phys. B342, 246 (1990).

[68] L. A. Pando Zayas and A. A. Tseytlin, 3-branes on resolved conifold, JHEP 0011, 028 (2000) arXiv:hep-th/0010088.

[69] I. R. Klebanov and A. Murugan, Gauge/Gravity Duality and Warped Resolved Conifold, JHEP 0703, 042 (2007) arXiv:hep-th/0701064.

[70] A. Giveon and D. Kutasov, Little string theory in a double scaling limit, JHEP 9910 034, (1999) [arXiv: hep-th/9909110

[71] D. Ghoshal and C. Vafa, $c=1$ string as the topological theory of the conifold, Nucl. Phys. B453 121, (1995) [arXiv: hep-th/9506122]

arXiv:hep-th/9511164.

[72] M. Shifman and A. Yung, Critical Non-Abelian Vortex in Four Dimensions and Little String Theory, Phys. Rev. D 96, no. 4, 046009 (2017) arXiv:1704.00825 [hep-th]].

[73] M. Shifman and A. Yung, to be published.

[74] Richard Feynman, The Character of Physical Law, ( MIT Press, 1965). 